Rev. Int. Contam. Ambie. 33 (1) 85-97, 2017

DOI: 10.20937/RICA.2017.33.01.08

\title{
CALIDAD DEL AGUA PARA RIEGO EN UNA ZONA NOGALERA DEL ESTADO DE CHIHUAHUA
}

\author{
María Cecilia VALLES-ARAGÓN ${ }^{1}$, Dámaris Leopoldina OJEDA-BARRIOS ${ }^{1 *}$, \\ Víctor Manuel GUERRERO-PRIETO ${ }^{1}$, Jesús Alejandro PRIETO-AMPARAN² y \\ Esteban SÁNCHEZ-CHÁVEZ ${ }^{3}$
}

${ }^{1}$ Facultad de Ciencias Agrotecnológicas, Universidad Autónoma de Chihuahua. Escorza 900, Colonia Centro 31000, Apartado Postal 24, Chihuahua, Chihuahua, México, C.P. 31000

${ }^{2}$ Facultad de Zootecnia, Universidad Autónoma de Chihuahua. Escorza 900, Colonia Centro 31000, Apartado Postal 24, Chihuahua, Chihuahua, México, C.P. 31000

${ }^{3}$ Centro de Investigación en Alimentación y Desarrollo S.C, Coordinación Delicias. Avenida 4ta. Sur 3820, Fraccionamiento Vencedores del Desierto, Ciudad Delicias, Chihuahua, México, C. P. 33089

*Autor para correspondencia: dojeda02@hotmail.com

(Recibido octubre 2015; aceptado mayo 2016)

Palabras clave: Carya illinoinensis, metales, $\mathrm{pH}$, nitratos, calidad del agua

\section{RESUMEN}

El cultivo de nogal pecanero es muy importante por su impacto económico en la región norte de México. La aplicación de agua en las huertas proveniente de pozos agrícolas con alto contenido de sales propicia su acumulación en el suelo, dificultando su movilidad hacia las raíces de los árboles. Con el propósito de analizar químicamente la composición del agua de riego en la región nogalera del sur de Chihuahua y contrastarla con las normas mexicanas vigentes se realiza la siguiente investigación. El muestreo se llevó a cabo el 25 de junio del 2014, se seleccionaron 10 huertas de nogal pecanero del sur del estado de Chihuahua y se tomó una muestra de agua de cada pozo. Los parámetros analizados fueron $\mathrm{pH}$, cloruros, $\mathrm{Fe}, \mathrm{Mn}, \mathrm{Zn}, \mathrm{Cu}, \mathrm{As}, \mathrm{B}, \mathrm{Pb}, \mathrm{Cd}, \mathrm{Ni}$, Na, sólidos disueltos totales, nitratos, dureza y alcalinidad, relación de absorción de sodio y conductividad eléctrica (CE). Para uso y consumo humano se consideró la Norma Oficial Mexicana 127, mientras que para riego agrícola se utilizó la Ley Federal de Derechos y Disposiciones Aplicables en Materia de Aguas Nacionales. Se obtuvieron valores dentro de los parámetros establecidos tanto para agua de consumo humano como en agua de riego en el sur del estado de Chihuahua, siendo dicha agua de buena calidad. $\mathrm{Sin}$ embargo, se sugiere tener un monitoreo periódico de las concentraciones de $\mathrm{Pb}, \mathrm{Ni}$, As, B y CE para evitar afectar a la población humana de comunidades circundantes en la región, así como impactos negativos en la actividad agrícola.

Key words: Carya illinoinensis, metalles, $\mathrm{pH}$, nitrates, water quality

\begin{abstract}
Cultivation of pecan nuts is very important for the economic impact in the northern region of Mexico. The application of water in the orchards from agricultural wells with high salt content, promotes their accumulation in the soil, hindering their mobility to tree roots. In order to chemically analyze the composition of water irrigation in the pecan-producing
\end{abstract}


region of southern Chihuahua and contrast it with the current Mexican standards, the following research was conducted. Sampling was carried out in June 25, 2014. Ten orchards in the south of the Chihuahua State were selected and a water sample from each well was taken. The analyzed parameters were $\mathrm{pH}$, chlorides, Fe, Mn, Zn, $\mathrm{Cu}, \mathrm{As}, \mathrm{B}$, $\mathrm{Pb}, \mathrm{Cd}, \mathrm{Ni}, \mathrm{Na}$, total dissolved solids, nitrates, hardness, alkalinity, sodium adsorption ratio and electric conductivity (EC). For human use and consumption was considered the Mexican official norm (NOM 127), and for agricultural irrigation the Federal Law of Rights - Provisions Applicable to Matters of National Waters. Values obtained in the south of the Chihuahua State were within the parameters established for both drinking and agricultural irrigation water, thus being this water of good quality. However, it is suggested to have a periodic monitoring of the concentrations of $\mathrm{Pb}, \mathrm{Ni}, \mathrm{As}, \mathrm{B}$ and $\mathrm{CE}$ to avoid the future possibility of repercussions to the human population of the surrounding communities in the region and of negative impacts on farming activity.

\section{INTRODUCCIÓN}

La superficie cultivada de nogal pecanero en México es de 98612 ha, se localiza en el norte del país, prácticamente en su totalidad en áreas de riego de gravedad y bombeo, donde participan los estados de Chihuahua con el $61.1 \%$, Coahuila con $16.2 \%$, Sonora con $9.7 \%$, Nuevo León con $4.3 \%$ y Durango con $6.1 \%$ (SIAP 2013).

Estas regiones presentan características edáficas y de clima que permiten el desarrollo óptimo del cultivo como son: clima seco semiárido, veranos calientes, amplios periodos de crecimiento superiores a los 200 días libres de heladas, con altas acumulaciones de calor durante el desarrollo de la nuez y suelos de neutros a alcalinos (Ojeda-Barrios et al. 2012). Las condiciones de altas tasas de evaporación y reducida precipitación pluvial, son factores que contribuyen a la acumulación de sales y sodio en el suelo, cuando se hace un manejo inadecuado del agua de riego con alto contenido de sales y prácticas de labranza inapropiadas en suelos arcillosos y con un deficiente drenaje en el perfil (Miyamoto 2004).

La aplicación de agua con alto contenido de sales hace que éstas se acumulen en el suelo, ya que la extracción de agua por el cultivo y la evaporación directa provoca que se concentren en las capas superficiales del suelo (Miyamoto y Nesbitt 2011). Esto hace que la humedad sea retenida en la superficie dificultando su movilidad hacia las raíces de los árboles. Si esto ocurre, pueden verse síntomas de deficiencia de agua como marchitez que significa cierre de estomas o reducción en la fotosíntesis, menor crecimiento de hojas, brotes y frutos aún cuando el suelo aparenta estar húmedo (Miyamoto 2015).

Aunado a esto, la excesiva aplicación de fertilizantes es una práctica estandarizada con poca consideración espacial, temporal y climática, lo que resulta en pérdida de ingresos y un impacto ambiental negativo (Sammis et al. 2013).

Numerosos estudios han demostrado que el uso de fertilizantes y pesticidas ha contribuido en gran medida a la mejora de la producción de alimentos. Sin embargo, el uso ineficiente de los mismos también puede dar lugar a un considerable riesgo a la salud humana. Principalmente para los agricultores y para el ambiente de ecosistemas agrícolas (Lu et al. 2015).

Relacionado a lo anterior la calidad del agua para riego es sumamente importante, ésta varía según el tipo y la cantidad de sales disueltas y a medida que el contenido aumenta, los problemas en el suelo y en los cultivos se incrementan (Valdez-Gascón et al. 2013).

Por demanda de los agricultores para conocer el estado actual de sus huertos de nogal en cuanto al contenido de sales, se realizó la presente investigación. Se determinaron parámetros físicos y químicos del agua utilizada para riego en huertos de nogal pecanero considerando: $\mathrm{pH}$, cloruros, nitratos, alcalinidad, dureza, solidos disueltos totales, relación de la absorción de sodio y conductividad eléctrica, así como la concentración de elementos inorgánicos $(\mathrm{Na}, \mathrm{Fe}, \mathrm{Mn}, \mathrm{Zn}, \mathrm{Cu}$, $\mathrm{As}, \mathrm{B}, \mathrm{Pb}, \mathrm{Cd}, \mathrm{y} \mathrm{Ni}$ ) que podrían estar presentes en los mantos acuíferos en el municipio de Jiménez, sureste del estado de Chihuahua. Además se compararon los resultados con los límites máximos permisibles (LMP) de la normatividad mexicana aplicable. De acuerdo con la NOM 127 SSA1-1994 (SSA 1994) para uso y consumo humano y la Ley Federal de Derechos y Disposiciones Aplicables en Materia de Aguas Nacionales (CONAGUA 2013) para riego agrícola.

\section{MATERIALES Y MÉTODOS}

Fueron seleccionadas 10 huertas de nogal pecanero en la carretera libre Jiménez-Camargo del estado 
de Chihuahua. Los pozos de los huertos escogidos se encuentran geoposicionados como se muestra en cuadro I. El municipio de Jiménez, ubicado hacia el este del estado de Chihuahua, cuyas condiciones climáticas y edafológicas son similares a las principales regiones productoras de nogal pecanero en el norte del país. La localidad se encuentra a $28^{\circ} 38^{\prime}$ latitud norte, $106^{\circ} 04^{\prime}$ longitud oeste, a una altitud de $1262 \mathrm{msnm}$, con $336.5 \mathrm{~mm}$ de precipitación anual. Es una región árida con una temperatura media anual de $18.6^{\circ} \mathrm{C}$ (Ojeda et al. 2012). Los suelos en los huertos se encuentran clasificados como xerosol cálcico (FAO/ UNESCO/ISRIC 1990), de textura variable pero preferentemente migajón arcilloso con un $\mathrm{pH}$ que fluctúan de 7.0 a 7.9 , materia orgánica de $0.87-1.09 \%$ que es medianamente bajo y con cantidades de $\mathrm{CaCO}_{3}$ de medias a altas (Ojeda-Barrios et al. 2012). Las plantaciones se encuentran en producción y tienen desde 15 hasta 35 años de edad, corresponden al cultivar Western Schley y Wichita injertados sobre patrón criollo. El sistema de plantación utilizado generalmente es marco real, a $12 \times 12 \mathrm{~m}$ con una densidad de 70 árboles/ha.

CUADRO I. GEOPOSICIÓN DE LOS POZOS DE AGUA DE RIEGO, MUESTREADOS POR LA CARRETERA JIMÉNEZ-LAS PAMPAS, CHIHUAHUA, MÉXICO 2015

\begin{tabular}{lccc} 
Huerta & $\begin{array}{c}\text { Número } \\
\text { de pozos }\end{array}$ & Latitud & Longitud \\
\hline $\begin{array}{l}\text { El Subsirio } \\
\text { Los Nogales }\end{array}$ & 1 & $27^{\circ} 18^{\prime} 08.71^{\prime \prime}$ & $104^{\circ} 51^{\prime} 40.16^{\prime \prime}$ \\
Ls Nogales & 1 & $27^{\circ} 16^{\prime} 24.37^{\prime \prime}$ & $104^{\circ} 49^{\prime} 38.25^{\prime \prime}$ \\
El Carmen: & 2 & $27^{\circ} 16^{\prime} 38.73^{\prime \prime}$ & $104^{\circ} 49^{\prime} 21.44^{\prime \prime}$ \\
El Desarrollo & 1 & $27^{\circ} 18^{\prime} 46.34^{\prime \prime}$ & $104^{\circ} 50^{\prime} 51.54^{\prime \prime}$ \\
El Carmen: & & & \\
El Desarrollo & 2 & $27^{\circ} 18^{\prime} 48.24^{\prime \prime}$ & $104^{\circ} 50^{\prime} 33.84^{\prime \prime}$ \\
El Carmen & 1 & $27^{\circ} 18^{\prime} 17.7^{\prime \prime}$ & $104^{\circ} 50^{\prime} 20.0^{\prime \prime}$ \\
El Carmen & 2 & $27^{\circ} 18^{\prime} 43.7^{\prime \prime}$ & $104^{\circ} 50^{\prime} 43.7^{\prime \prime}$ \\
El Faro & 1 & $27^{\circ} 18^{\prime} 47.5^{\prime \prime}$ & $104^{\circ} 51^{\prime} 59.1^{\prime \prime}$ \\
La Concha & 1 & $27^{\circ} 13^{\prime} 31.90^{\prime \prime}$ & $104^{\circ} 46^{\prime} 7.53^{\prime \prime}$ \\
La Trinidad & 1 & $27^{\circ} 17^{\prime} 31.4^{\prime \prime}$ & $104^{\circ} 49^{\prime} 51.4^{\prime \prime}$ \\
\hline
\end{tabular}

Previa autorización de los propietarios, se acordó recolectar las muestras entre las 6:00 y 7:30 am del 25 de junio de 2014. Los pozos se pusieron a trabajar una hora antes de recolectar la muestra. En el cuadro I se encuentran los pozos de agua de uso agrícola seleccionados para este trabajo de investigación y su ubicación geográfica. El agua se recolectó en envases de plástico esterilizados de un L, los cuales estaban previamente identificados. Una vez recolectadas las muestras se pusieron en una hielera para mantener estable la temperatura.

Dichas muestras fueron llevadas a la ciudad de Delicias, Chihuahua al Laboratorio del Centro de Investigación en Alimentación y Desarrollo (CIAD) para su posterior análisis. Al llegar al CIAD se determinaron los valores de $\mathrm{pH}$ y conductividad eléctrica, inmediatamente después se refrigeraron para la evaluación de los demás parámetros evaluados en este estudio.

Los parámetros considerados y métodos de análisis fueron: $\mathrm{pH}$-potenciómetro, cloruros $\left(\mathrm{Cl}^{-}\right)$-titulación, sólidos disueltos totales (SDT)-gravimétrico, conductividad eléctrica (CE)-conductímetro. Asimismo, fueron determinados por espectrofotometría de absorción atómica: fierro (Fe), manganeso $(\mathrm{Mn})$, zinc $(\mathrm{Zn})$, cobre $(\mathrm{Cu})$, arsénico (As), boro (B), plomo $(\mathrm{Pb})$, cadmio $(\mathrm{Cd})$ y níquel $(\mathrm{Ni})$. Estos parámetros se compararon con los LMP de la normatividad mexicana aplicable. Para uso y consumo humano se consideró la NOM 127 SSA1-1994 (SSA 1994) y para riego agrícola la Ley Federal de Derechos y Disposiciones Aplicables en Materia de Aguas Nacionales (CONAGUA 2013). Simultáneamente, se determinaron otros parámetros regulados sólo para agua de uso y consumo humano (SSA 1994) como: nitratos-espectrofotometría, sodio $(\mathrm{Na})$-espectrofotometría de absorción atómica, dureza y alcalinidadtitulación. Además, fue cuantificada la relación de absorción de sodio (RAS), regulada sólo para riego agrícola (CONAGUA 2013). También se realizó en la zona de estudio un mapa de la distribución de As, Ni y B a través del programa ArcGIS, versión 10.1.

\section{RESULTADOS Y DISCUSIÓN}

\section{pH del agua de riego}

El $\mathrm{pH}$ en agua aceptable para uso y consumo humano ya que se encuentra en un rango de 6.5 a 8.5 (SSA 1994), mientras que para el agua de riego agrícola va de 6.0 a 9.0 (CONAGUA 2013). El pH de los pozos muestreados estuvo en el rango de $7.2 \mathrm{a}$ 8.0, clasificado como ligeramente alcalino, dentro de lo permitido en la normatividad tanto para consumo humano como para riego agrícola. Este parámetro químico se considera uno de los más necesarios para indicar la calidad del agua potable (Rajendran y Mansiya 2015), el cual varía debido a los diferentes gases y sólidos disueltos en el agua (Patil et al. 2012).

\section{Cloruros}

La mayoría de las concentraciones de cloruros de los pozos analizados estuvieron dentro de los LMP 
de $7.0 \mathrm{meq} / \mathrm{L}$ para agua de consumo humano y 4.2 $\mathrm{meq} / \mathrm{L}$ para agua de riego agrícola, establecidos por la normatividad mexicana. El pozo ubicado en el Carmen Desarrollo 1, superó los valores de referencia para uso y consumo humano y para riego agrícola (Fig. 1; SSA 1994, CONAGUA 2013). Los cloruros están presentes en todas las aguas naturales en concentraciones variables. La magnitud es mayor en aguas subterráneas que en superficiales, especialmente si hay depósitos de sal en el área (Páez-Sánchez et al. 2013).

La alta concentración de estos aniones puede ser debido a la presencia de la piedra caliza, dolomita, yeso, sales y carbones dentro de los sedimentos cuaternarios (Arain et al. 2014). Altas concentraciones de cloruros pueden evitar el crecimiento de las plantas y causar daños a estructuras metálicas, como causar la corrosión de tuberías, lo que puede ser potencialmente peligroso si dichas tuberías están compuestas de metales tóxicos (Páez-Sánchez et al. 2013). Los iones cloruro en altas concentraciones son conocidos por ser perjudiciales para cítricos y cultivos leñosos. Un contenido de cloruro mayor a $10 \mathrm{meq} / \mathrm{L}$ puede causar problemas graves a los cultivos (Phocaides 2000).

\section{Sólidos disueltos totales (SDT)}

Todas las muestras de agua para riego agrícola superaron los niveles de referencia $(500 \mathrm{mg} / \mathrm{L})$ de SDT (CONAGUA 2013). Para agua de uso y consumo humano sólo dos pozos estuvieron por debajo del nivel de referencia de $1000 \mathrm{mg} / \mathrm{L}$ (SSA 1994),
Los Nogales 2 y el Carmen 2 (Fig. 2). Los SDT es la medida del contenido de todas las sustancias inorgánicas y orgánicas en un líquido en forma suspendida molecular, ionizada o micro granulada (Arain et al. 2014). Altas concentraciones de SDT, como es el caso de estos pozos, sugieren la presencia de sales inorgánicas, principalmente $\mathrm{Ca}, \mathrm{Mg}, \mathrm{K}, \mathrm{Na}$, bicarbonatos, sulfatos y cloruros (Patil et al. 2012, Páez-Sánchez et al. 2013).

\section{Fierro, manganeso, zinc y cobre}

Para agua de uso y consumo humano los LMP de $\mathrm{Fe}, \mathrm{Mn}, \mathrm{Zn}$ y Cu son $0.3,0.15,5.0$ y $2.0 \mathrm{mg} / \mathrm{L}$, respectivamente (SSA 1994). Mientras que los LMP de Fe, $\mathrm{Mn}, \mathrm{Zn}$ y Cu para agua de riego agrícola son 5.0, 0.2, 2.0 y $0.2 \mathrm{mg} / \mathrm{L}$, respectivamente (CONAGUA 2013). Las concentraciones de todos los pozos de muestreo estuvieron debajo de los LMP por la normatividad aplicable (Fig. 3). Estos elementos (Fe, Mn, $\mathrm{Zn}$ y Cu) se consideran nutrientes esenciales para el desarrollo óptimo del nogal (Ojeda-Barrios et al. 2014). Sin embargo, a altas concentraciones en el agua pueden tener efectos nocivos en otros cultivos y seres vivos, por ello su monitoreo (Ayers y Westcot 1985).

El Fe es un elemento que se encuentra en grandes cantidades en la corteza terrestre. No obstante, para el nogal es un elemento no disponible en suelos calcáreos que impiden su absorción y traslocación (Vargas Piedra y Arreola Ávila 2008). En general es un metal importante en plantas y animales, sobre todo

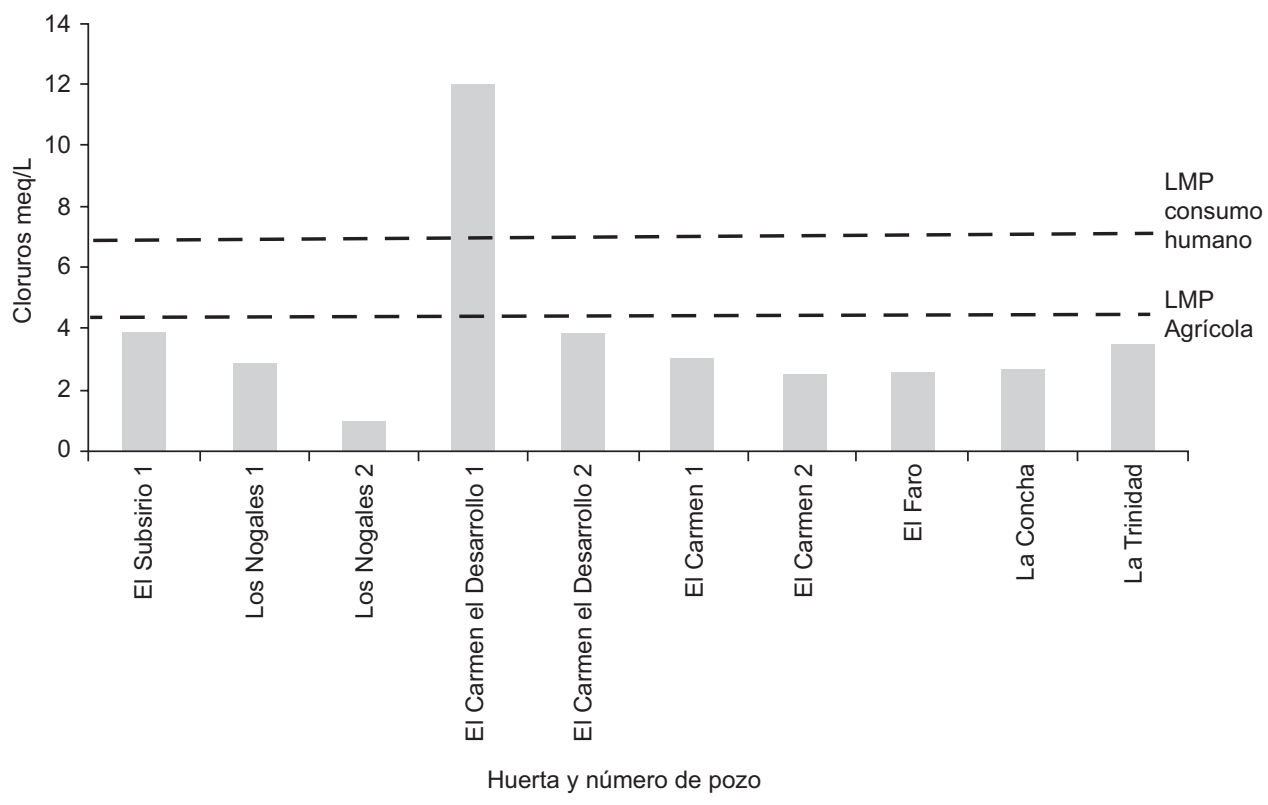

Fig. 1. Concentración de cloruros en agua de los pozos muestreados y límites máximos permisibles (LMP) en México para consumo humano y riego agrícola 


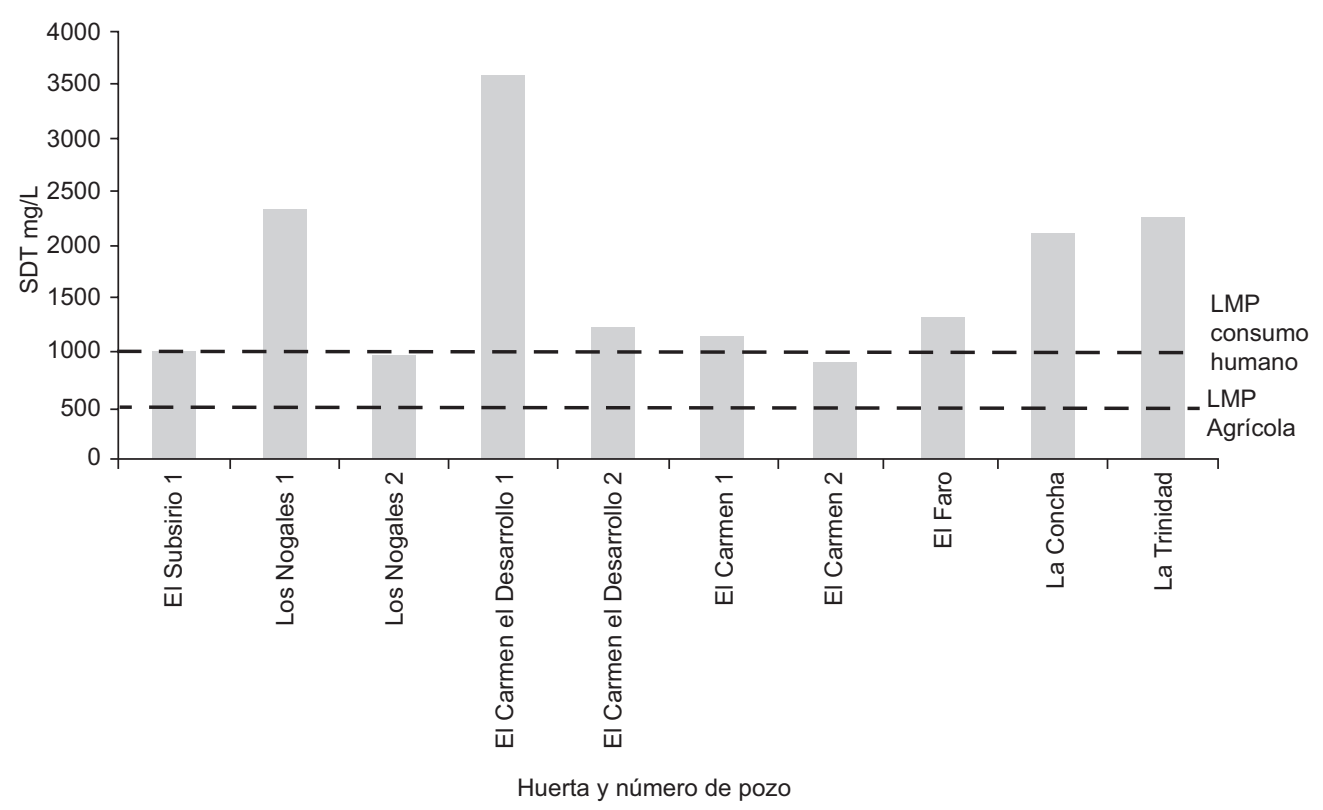

Fig. 2. Sólidos disueltos totales (SDT) analizados en los pozos de muestreo y límites máximos permisibles (LMP) en México para consumo humano y riego agrícola

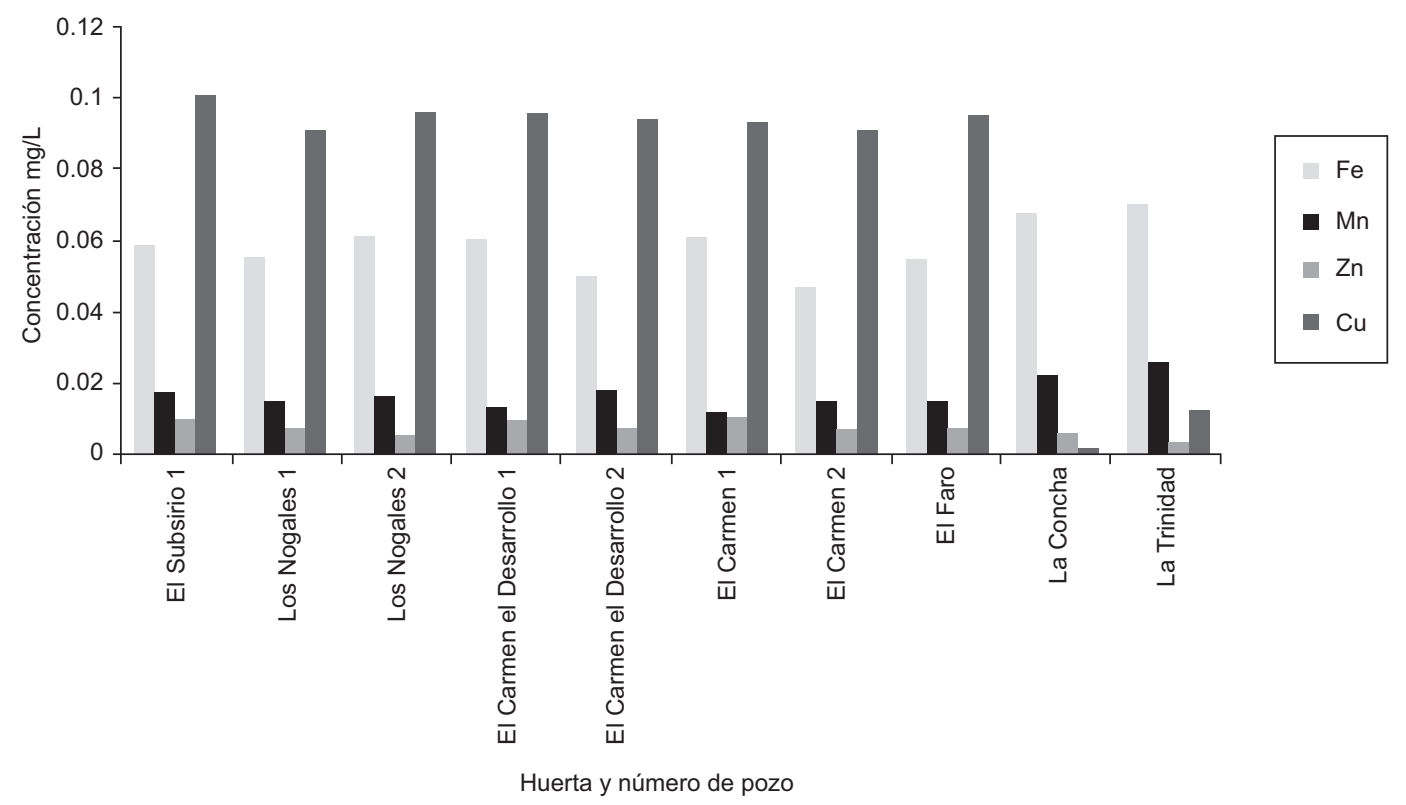

Fig. 3. Concentración de fierro $(\mathrm{Fe})$, manganeso $(\mathrm{Mn})$, zinc $(\mathrm{Zn})$ y cobre $(\mathrm{Cu})$ en agua de pozo de la zona muestreada

por su participación activa en la síntesis del material genético de las células (Arain et al. 2014). Además, no es tóxico para las plantas, pero puede contribuir a la acidificación del suelo y a la pérdida de disponibilidad de fósforo y molibdeno (Ayers y Westcot 1985).

El Mn está ampliamente involucrado en procesos catalíticos, siendo el nutrimento activador en algunas enzimas en la respiración, en la asimilación de nitrógeno y en la fotosíntesis (Vargas Piedra y Arreola Ávila 2008).

El Zn no es absorbido por las raíces del nogal en suelos alcalinos con alto contenido de $\mathrm{Ca}$ (como existen de manera predominante en el norte de México donde se cultiva el nogal). Por lo tanto, el abastecimiento de $\mathrm{Zn}$ por vía foliar es indispensable, lo que requiere realizar aspersiones foliares cada año 
(Ojeda-Barrios et al. 2012). Asimismo, el Zn es tóxico para muchas otras plantas a concentraciones que varían ampliamente, su toxicidad se reduce a $\mathrm{pH}>6$ y en textura fina o suelos orgánicos (Patil et al. 2012).

El Cu se considera tóxico para una serie de plantas de 0.1 a $1.0 \mathrm{mg} / \mathrm{L}$ en soluciones nutritivas (Ayers y Westcot 1985). Sin embargo, para el nogal, el Cu es necesario en cantidades extremadamente pequeñas (Vargas Piedra y Arreola Ávila 2008).

\section{Arsénico}

La mayoría de los pozos de muestreo para riego agrícola quedaron dentro del LMP de As $(0.1 \mathrm{mg} / \mathrm{L}$; CONAGUA 2013), excepto Los Nogales 1 y La Trinidad. Varios pozos excedieron dichos límites de As para agua de uso y consumo humano (0.025 mg/L; SSA 1994). Entre ellos, Los Nogales 1, La Trinidad, el Carmen Desarrollo 2 y el Carmen 1 (Fig. 4 y Fig. 5).

Resulta relevante que el As ha sido detectado en varias fuentes de agua potable en México. Chihuahua es una de las zonas más afectadas por su presencia de manera natural en agua subterránea. Hay estudios que evidencian la problemática de contaminación en fuentes de agua potable con As en la región sureste del estado de Chihuahua, incluyendo el municipio de Jiménez donde un alto porcentaje de pozos, presentan valores de As por encima de la NOM 127 en más del $50 \%$ de sus fuentes de abastecimiento (Olmos-Márquez 2011).

Debido a que el As es un elemento no esencial para el desarrollo de los seres humanos, se considera un elemento potencialmente tóxico de alta prioridad (Bauer et al. 2008). Asimismo, también resulta nocivo para el crecimiento adecuado de plantas y cultivos (García 2012).

\section{Boro}

Los pozos el Carmen 2 y la Concha excedieron los valores de referencia de $\mathrm{B}$, tanto en agua para riego agrícola $(0.7 \mathrm{mg} / \mathrm{L})$ (CONAGUA 2013), como para uso y consumo humano $(1.0 \mathrm{mg} / \mathrm{L})(\mathrm{SSA} 1994$; Fig. 5 y Fig. 6).

El B es un elemento esencial para el crecimiento de las plantas, pero en cantidades excesivas se vuelve tóxico (Bañuelos et al. 1999), incluso en concentraciones relativamente bajas de $0.6 \mathrm{mg} / \mathrm{L}$ (Phocaides 2000). Concentraciones tóxicas son comunes en áreas donde las aguas subterráneas que se usan para riego atraviesan depósitos ricos en este nutrimiento (García 2012). En las regiones áridas, el B es considerado el elemento más perjudicial en el agua de riego (Phocaides 2000).

El B puede ser absorbido por las raíces junto con el agua, acumulándose en las hojas y otras partes de las plantas. Los síntomas típicos se representan en la punta de las hojas más antiguas que amarillean o presentan sequedad en los tejidos. Cuando los árboles están seriamente afectados, a veces producen exudación en troncos y ramas (Bañuelos et al. 1999). Los nogales son muy sensibles a la presencia de B (Wilcox y Durum 1967, Rhoades y Loveday 1990, Wells et al. 2008).

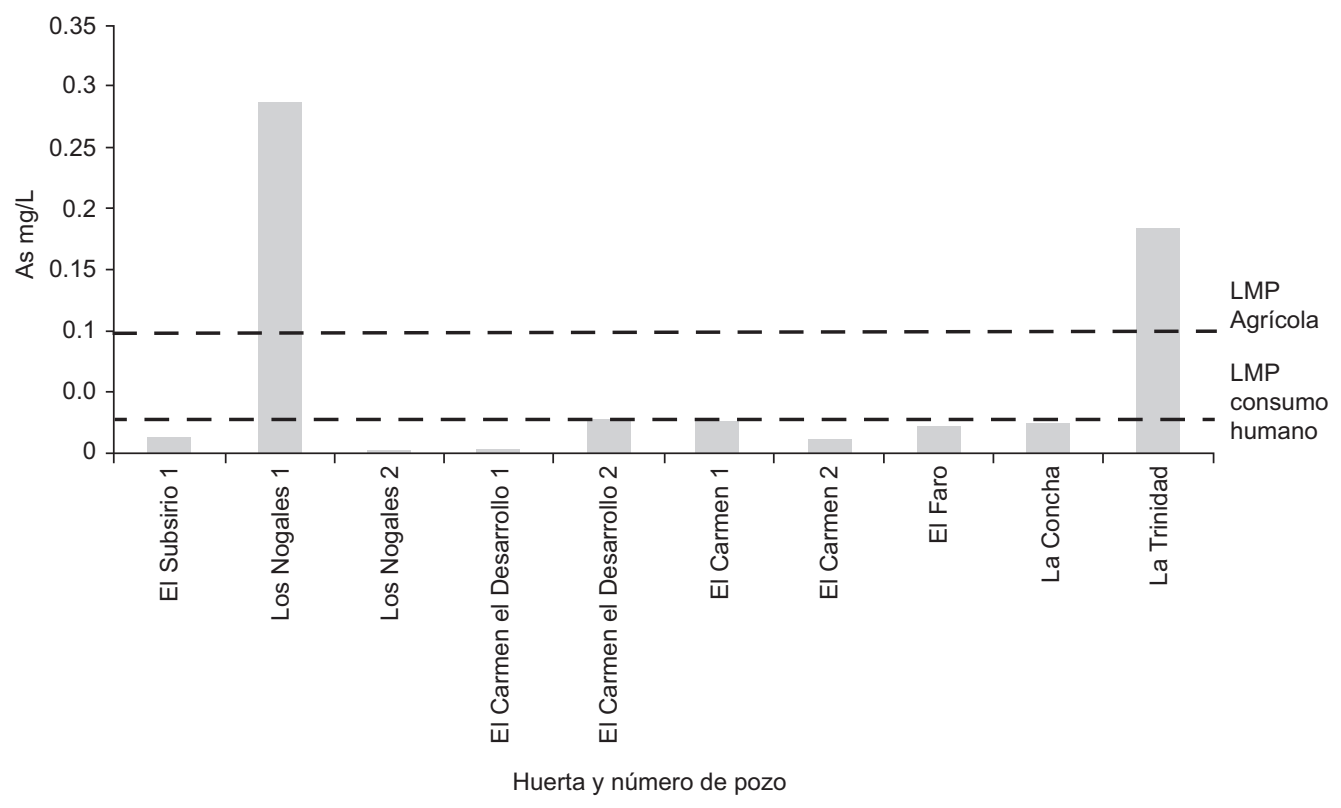

Fig. 4. Concentración de arsénico (As) en agua de pozo del área muestreada y límites máximos permisibles (LMP) en México para consumo humano y riego agrícola 

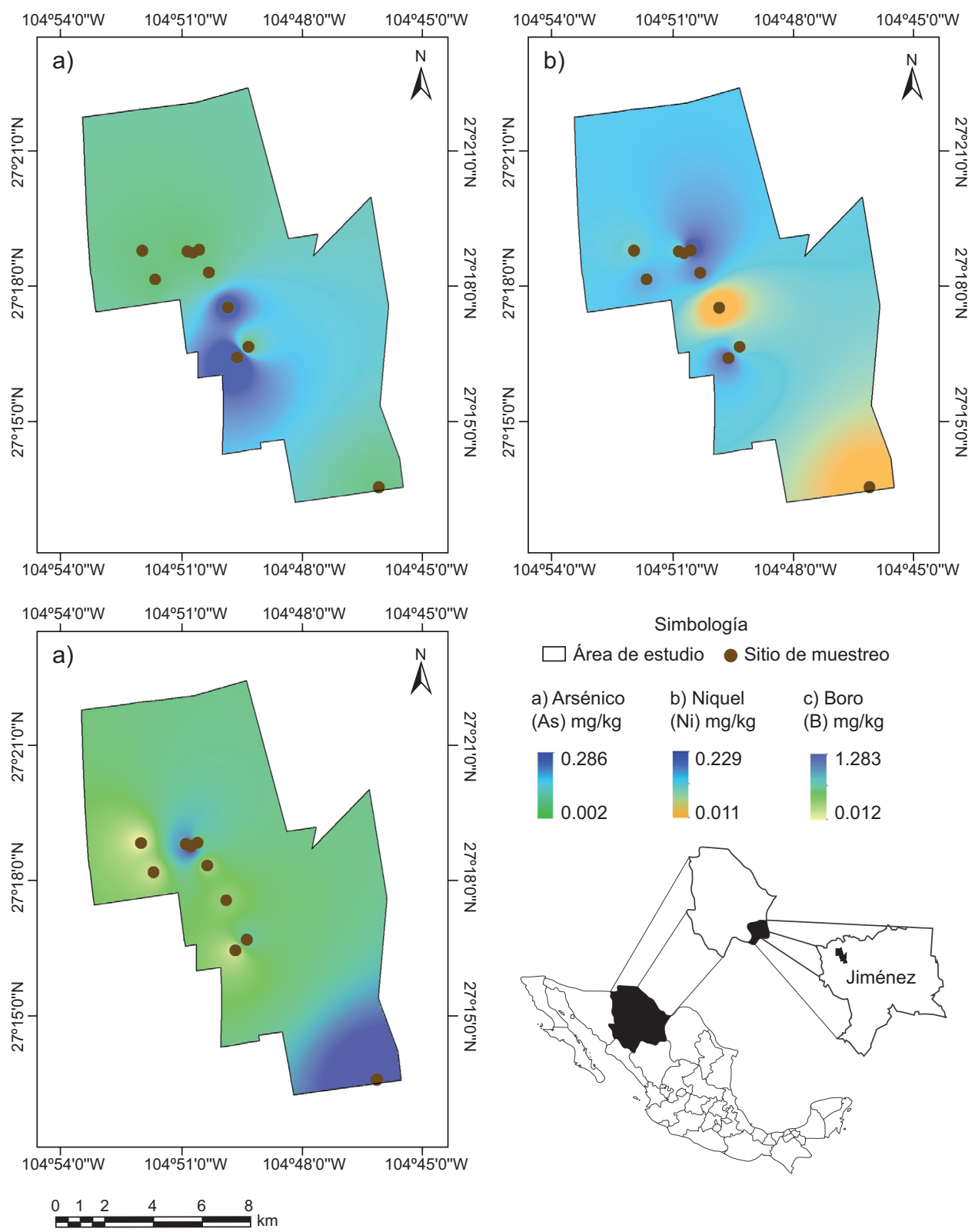

$\square$ Área de estudio o Sitio de muestreo
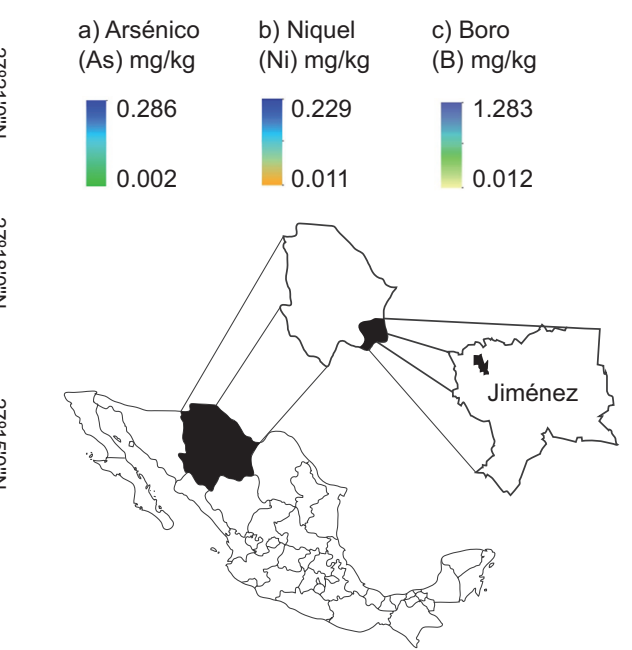

Fig. 5. Distribución de arsénico (As), níquel (Ni) y boro (B) en la zona de estudio

\section{Plomo}

Las concentraciones de $\mathrm{Pb}$ en todos los pozos de muestreo estuvieron dentro de los valores de referencia para agua de uso agrícola $(0.5 \mathrm{mg} / \mathrm{L})(\mathrm{CONAGUA}$ 2013). Las muestras de el Carmen 2, el Carmen 1 y la Concha, excedieron los LMP para uso y consumo humano (0.01 mg/L; SSA 1994; Fig. 7).

Aunque dos pozos superan los niveles permitidos para consumo humano, se destaca que el $\mathrm{Pb}$ no es requerido para el funcionamiento normal de los seres vivos, e incluso resulta tóxico para su desarrollo. Debido a su tamaño y carga, el $\mathrm{Pb}$ puede sustituir al Ca y acumularse en los huesos o intervenir en los procesos de los seres vivos que son dependientes del Ca (ATSDR 2005). Además, el $\mathrm{Pb}$ puede inhibir el crecimiento de células vegetales a concentraciones muy altas (Ayers y Westcot 1985, García 2012).

\section{Cadmio}

En todos los pozos muestreados los niveles de Cd estuvieron dentro de los LMP por la normatividad para uso y consumo humano $(0.005 \mathrm{mg} / \mathrm{L}$; SSA 1994) y para riego agrícola $(0.1 \mathrm{mg} / \mathrm{L}$; CONAGUA 2013; Fig. 7). El Cd es un metal muy tóxico a bajas 


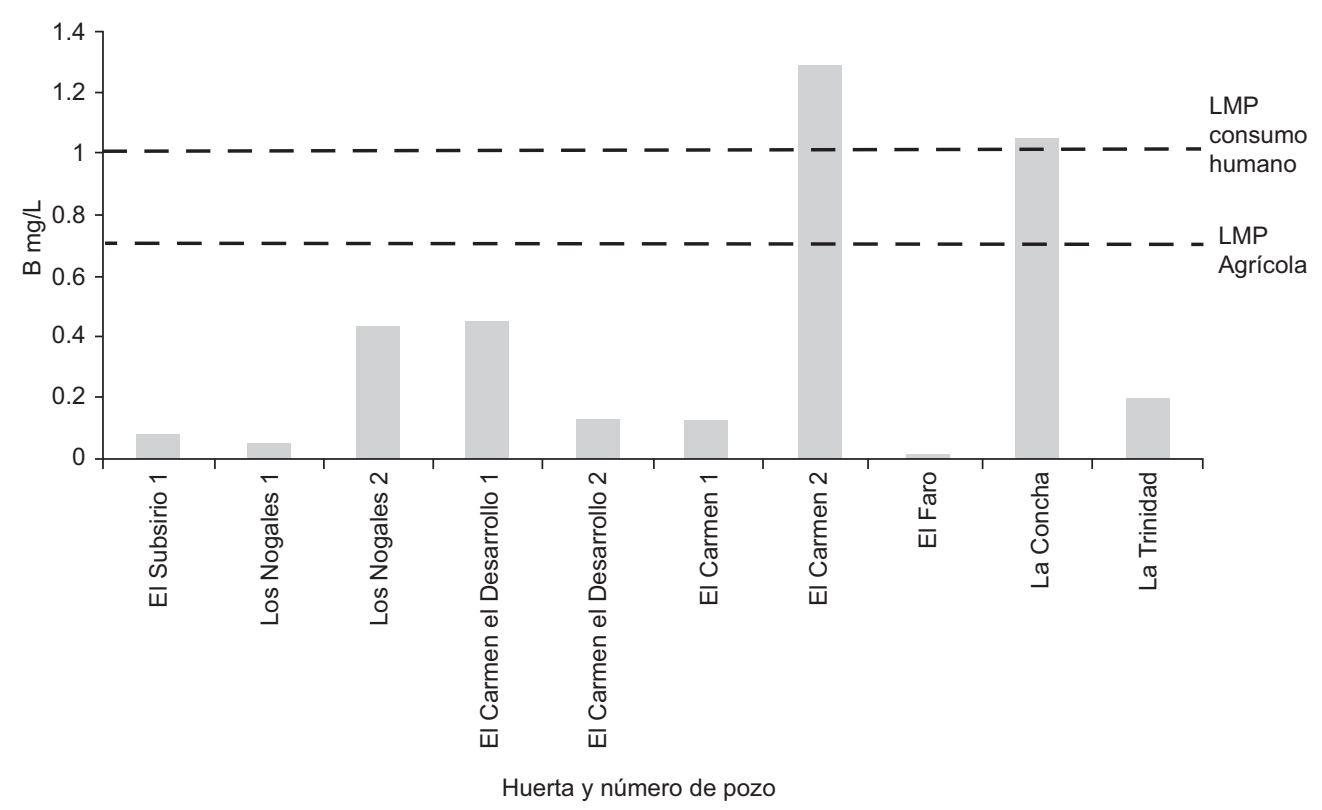

Fig. 6. Concentración de boro (B) en agua de los pozos muestreados y límites máximos permisibles (LMP) en México para consumo humano y riego agrícola

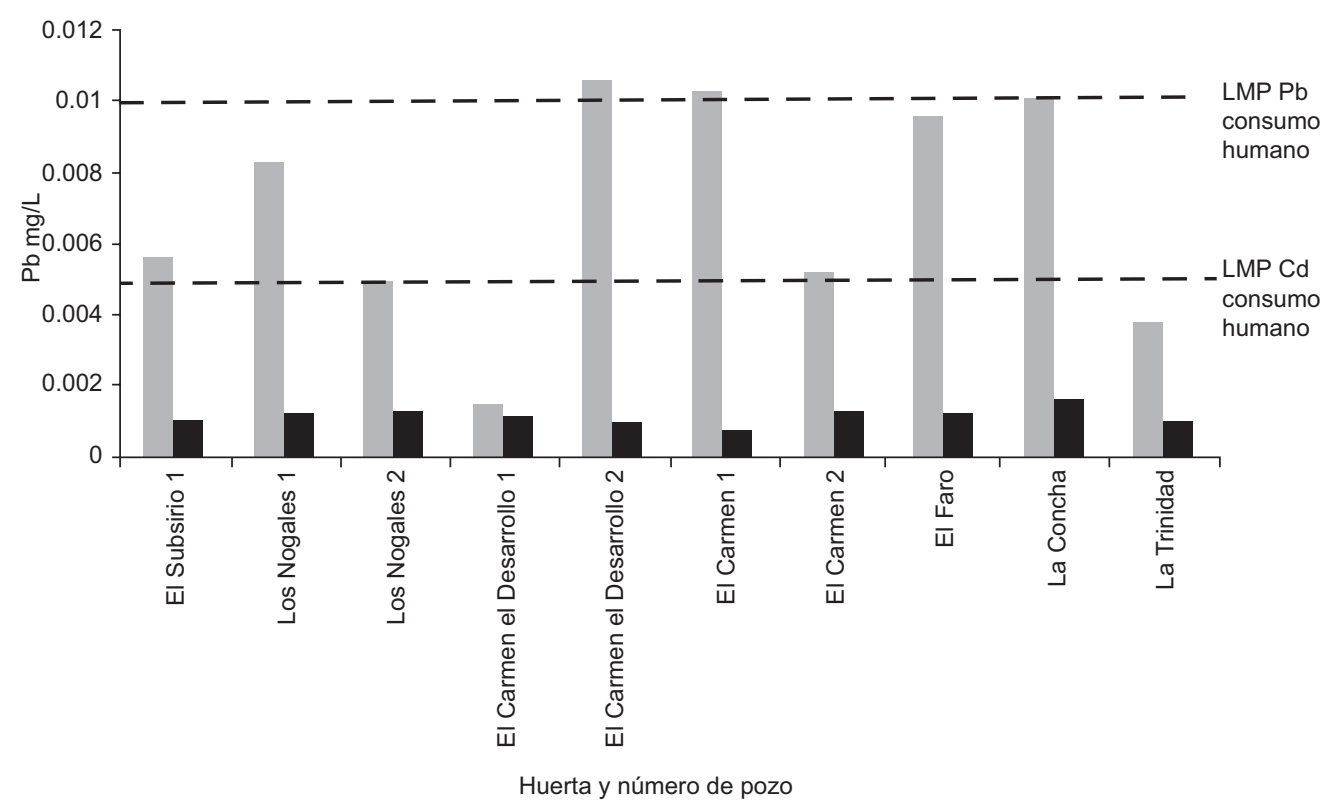

Fig. 7. Concentración de plomo $(\mathrm{Pb})$ y cadmio $(\mathrm{Cd})$ en agua de los pozos de muestreo y límites máximos permisibles (LMP) en México para consumo humano

concentraciones, por lo cual se recomiendan límites conservadores debido a su potencial de acumulación en plantas y suelos a concentraciones que pueden ser perjudiciales para los seres humanos (Ayers y Westcot 1985).

\section{Níquel}

En el caso de este elemento, se sobrepasaron los LMPde Ni en agua para consumo humano de $0.01 \mathrm{mg} / \mathrm{L}$
(SSA 1994) en los pozos evaluados. Sin embargo, ninguno excedió el nivel establecido para riego agrícola de $0.20 \mathrm{mg} / \mathrm{L}$ (CONAGUA 2013) (Fig. 8).

En la figura 5 se observa la distribución de la concentración de Ni en el área de estudio.

En la actualidad hay investigaciones que sustentan que el nogal requiere de 17 elementos esenciales para su buen desarrollo entre estos el Ni (Wood 2004 a y b, Alanís-Fierro 2014). Adicionalmente, se asegura 


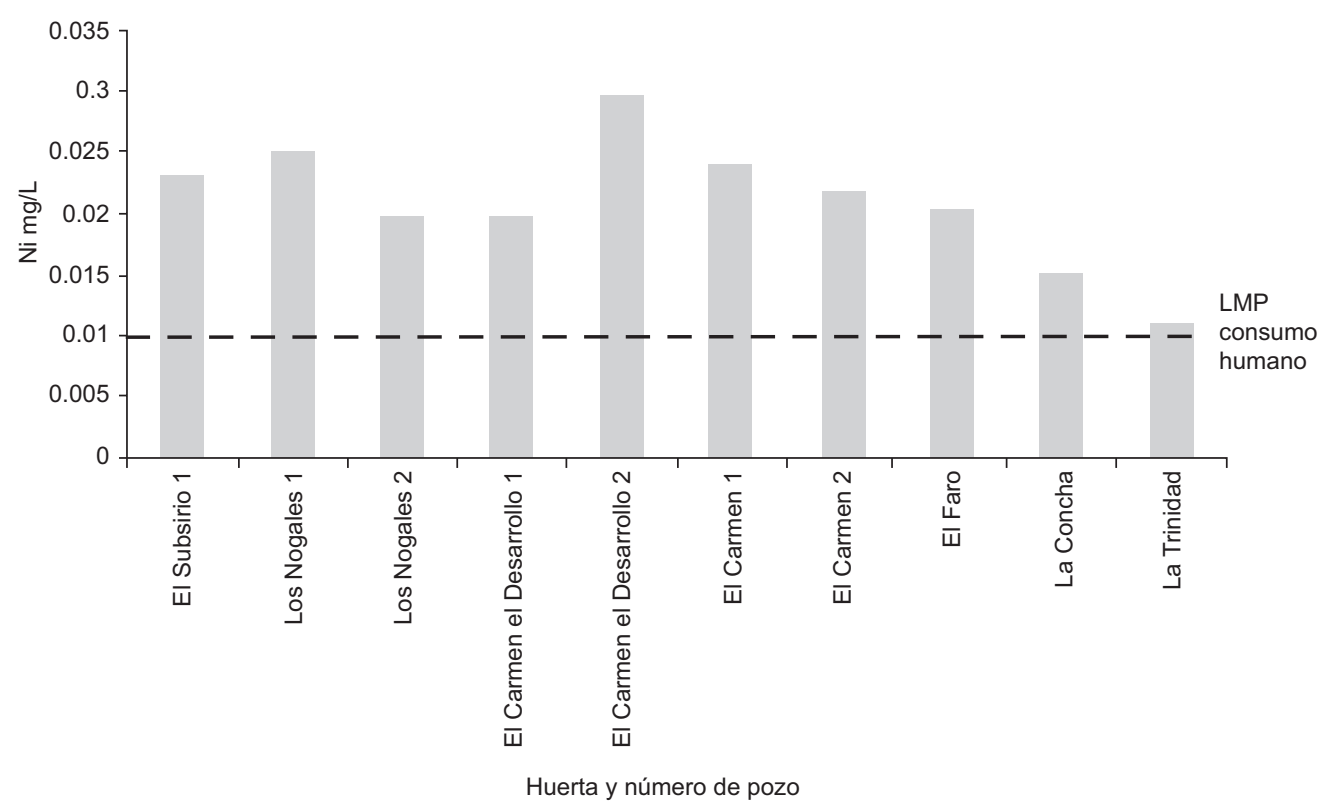

Fig. 8. Concentración de níquel (Ni) en pozos de muestreo y límite máximo permisible (LMP) en México para consumo humano

que la enfermedad de oreja de ratón o el trastorno en árboles de nogal pecanero son causados por una deficiencia de $\mathrm{Ni}$, que se subsana con una aplicación foliar oportuna (Wood 2006).

Investigaciones demuestran que la acumulación excesiva de metales en suelo por el uso de fertilizantes a largo plazo resulta una de las causas primarias de la deficiencia de Ni. Por lo que se plantea la posibilidad de que el $\mathrm{Ni}$ es un factor que contribuye a contrarrestar ciertas enfermedades recalcitrantes de determinados cultivos perennes leñosos. Por tal razón se considera que la actuación del nutriente, es como un elemento esencial (Wood 2006, Wood y Grauke 2011).

Brotes de nogal desarrollaron tejido necrótico en el extremo basal de los frutos en un huerto que recibió grandes cantidades de $\mathrm{N}$ de agua de riego contaminada con nitratos. Se planteó que el aumento de $\mathrm{Ni}$ y $\mathrm{Cu}$ podría mitigar los efectos negativos del exceso de $\mathrm{N}$ y reducir la incidencia de necrosis del núcleo (Wagle 2011).

Por todo lo anterior, se considera la posibilidad de que los mantos acuíferos de la zona de estudio están siendo perjudicados por la adición de Ni debido a las actividades agrícolas productoras de nuez. Lo cual estará además afectando a las comunidades humanas de la región por la ingesta de este metal en el agua de consumo en un futuro, entre otras interacciones que se pueden establecer en la misma cadena trófica.

\section{Nitratos, sodio, dureza, alcalinidad}

Los nitratos, sodio, dureza y alcalinidad quedaron dentro de los LMP para agua de uso y consumo humano. Los nitratos se permiten hasta $10 \mathrm{mg} / \mathrm{L}$, el $\mathrm{Na} 200 \mathrm{mg} / \mathrm{L}$, la dureza $500 \mathrm{mg} / \mathrm{L}$ y la alcalinidad $400 \mathrm{mg} / \mathrm{L}$ (Fig. 9). El monitoreo de estos parámetros en agua es relevante debido a los diversos efectos que pueden tener fuera de los LMP, tanto para irrigación como para uso y consumo humano, además pueden resultar un indicador de infiltración de contaminantes a la fuente de abasto.

La alta presencia de nitratos en agua puede deberse a la infiltración resultante del uso de fertilizantes, fugas de tanques sépticos, descarga de aguas residuales o erosión de los recursos naturales (Patil et al. 2012). El nitrógeno en el agua de riego es generalmente beneficioso para la mayoría de cultivos, pero puede causar problemas en algunos de ellos como, el betabel (o remolacha dulce), uva, chabacano, cítricos y aguacate (Ayers 1994).

El Na es un componente natural del agua (Patil et al. 2012), sin embargo en grandes cantidades puede causar daño directo o indirecto a muchas plantas (Phocaides 2000). Síntomas de toxicidad típicos son quemaduras en las hojas, necrosis prematura y tejido muerto a lo largo de los bordes exteriores de las hojas en contraste con los síntomas de la toxicidad (Ayers 1994).

La dureza en todos los pozos se encuentra por debajo de $75 \mathrm{mg} / \mathrm{L}$ lo que clasifica como agua suave 


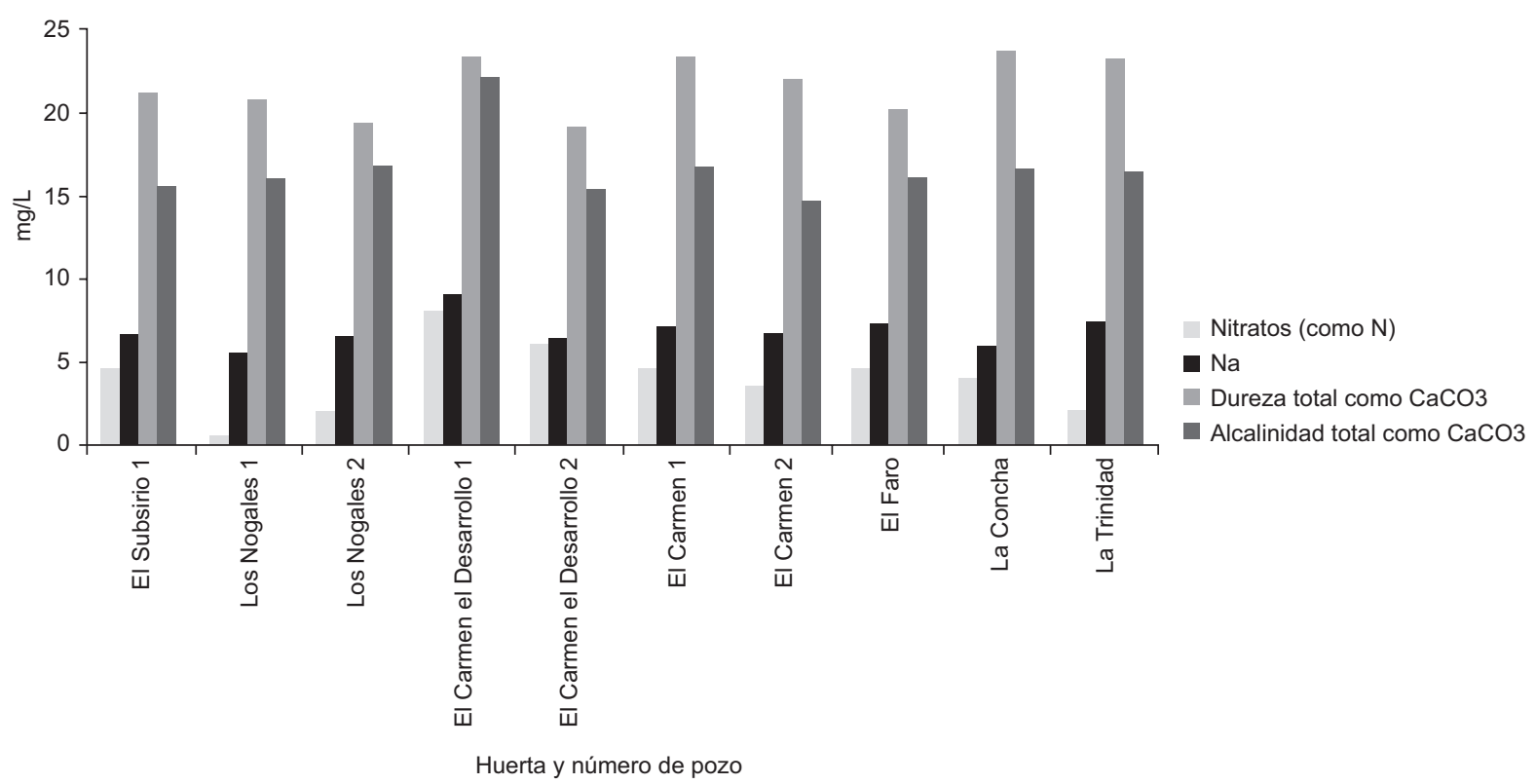

Fig. 9. Concentración de nitratos $(\mathrm{N})$, sodio $(\mathrm{Na})$, dureza y alcalinidad en agua de pozo muestreada

(Gebreyohannes et al. 2015), esto significa que el agua tiene alta capacidad de amortiguamiento, por lo que no puede generar tuberías corroídas y con ello contaminación externa del agua (Arain et al. 2014). Un agua dura se considera no adecuada para uso industrial y agrícola (Patil et al. 2012).

Los valores altos de alcalinidad podrían conducir a un sabor amargo y a una alta salinidad en dicha agua (Páez-Sánchez et al. 2013).

Relación de absorción de sodio en agua y conductividad eléctrica

La normatividad regula cuatro puntos para la RAS en agua. Los pozos analizados quedaron dentro de este límite (Cuadro II). El RAS por debajo de 10 representa un agua útil para riego, sin embargo, valores por debajo de 5 son deseables (Valdez-Gascón et al. 2013).

De los sistemas más desarrollados para alertar sobre el peligro de salinización o modificación del suelo a partir de algunos parámetros medidos en agua de riego, la conductividad eléctrica del agua es la medida ideal (Pérez-León 2011).

En relación con el diagrama de clasificación del agua citado en la investigación de Pérez-León (2011), el valor de RAS en todas las muestras está dentro de la clasificación de bajas en $\mathrm{Na}$. Considerando la CE, el agua de estos pozos se clasifica como de alta salinidad y de muy alta salinidad, mientras que el agua de el Carmen Desarrollo 1 quedó clasificada con salinidad excesiva. Asimismo, según el diagrama, al agua de cada pozo (C3-S1, C4-S1, C5-S1) se clasifica como utilizable para riego con precauciones (Cuadro II). Por lo que, se sugiere tener cuidado en la selección de cultivos que se riegan con dicha agua.

La CE alta y los elevados niveles de SDT están correlacionados (0.999), lo que indica la disolución de minerales (Patil et al. 2012). Los SDT altos pueden generar el aumento en la salinidad o cambios en la composición del agua (Gebreyohannes et al. 2015). Asimismo, la CE en aguas naturales está influenciada por las sales disueltas, tales como cloruro sódico y cloruro potásico (Arain et al. 2014). Lo anterior concuerda con los resultados obtenidos, pues el pozo con mayor concentración de $\mathrm{Cl}^{-}$y mayor cantidad de SDT es el mismo que presenta la más alta concentración de $\mathrm{CE}$ y ha sido clasificado como excesivamente salino (el Carmen Desarrollo 1). Lo cual resulta relevante en esta investigación pues el nogal pecanero, es sensible a las sales y a la presencia de cloruros (Grageda Grageda et al. 2011).

El agua salina puede provocar menos cantidad de agua disponible para las plantas, a pesar de que el suelo esté húmedo. Esto es porque las plantas sólo transpiran agua "pura" lo que puede disminuir el potencial del riego (Gebreyohannes et al. 2015). La salinidad es un problema común que enfrentan los agricultores en climas áridos. Si la precipitación pluvial es baja $(<380 \mathrm{~mm}$ anuales), el riego del terreno introduce sales y al evaporarse el agua de la 
CUADRO II. COMPARACIÓN DE LA RELACIÓN DE ABSORCIÓN DE SODIO (RAS) Y LA CONDUCTIVIDAD ELÉCTRICA (CE) EN LOS POZOS DE MUESTREO

\begin{tabular}{c|c|c|c|c|c}
\hline Huerta & RAS & $\begin{array}{c}\text { Clasificación } \\
\text { Sodio }\end{array}$ & CE (dS/m) & $\begin{array}{c}\text { Clasificación } \\
\text { Salinidad }\end{array}$ & $\begin{array}{c}\text { Clasificación } \\
\text { Del agua }\end{array}$ \\
\hline El Subsirio 1 & 1.03 & Baja & 1.57 & Alta & C3-S1 \\
Los Nogales 1 & 0.86 & Baja & 3.63 & Muy Alta & C4-S1 \\
Los Nogales 2 & 1.05 & Baja & 1.49 & Alta & C3-S1 \\
El Carmen El Desarrollo 1 & 1.38 & Baja & 5.61 & Excesiva & C5-S1 \\
El Carmen El Desarrollo 2 & 1.05 & Baja & 1.95 & Alta & C3-S1 \\
El Carmen 1 & 1.05 & Baja & 1.81 & Alta & C3-S1 \\
El Carmen 2 & 1.01 & Baja & 1.42 & Alta & C3-S1 \\
El Faro & 1.15 & Baja & 2.09 & Alta & C3-S1 \\
La Concha & 0.86 & Baja & 3.29 & Muy Alta & C4-S1 \\
La Trinidad & 1.09 & Baja & 3.5 & Muy Alta & C4-S1 \\
\hline
\end{tabular}

superficie del suelo, dichas sales permanecen ahí, lo que provoca la acumulación gradual de las mismas en la zona radicular (Phocaides 2000).

\section{CONCLUSIONES}

Se obtuvieron valores dentro de los parámetros establecidos para ambos usos de agua en las variables de $\mathrm{pH}$, cloruros, nitratos, dureza, alcalinidad, $\mathrm{Fe}, \mathrm{Mn}, \mathrm{Zn}, \mathrm{Cu}, \mathrm{Cd}$, Na y RAS. Sin embargo, se encontraron otras variables que sobrepasan los LMP para consumo humano como $\mathrm{Pb}$ y $\mathrm{Ni}$, que sin embargo, se encontraron dentro del rango permisible para uso agrícola. Por otro lado, las determinaciones de As, B y SDT sobrepasaron los LMP para consumo humano y agua de riego en algunos de los pozos de muestreo. En la relación de RAS con CE, el agua se clasificó con alta salinidad y muy alta salinidad, pero debido a su bajo contenido de $\mathrm{Na}$ se puede usar para riego de nogal pecanero. Sólo un pozo se clasifica como excesivamente salino, en el cual es necesario tomar medidas para mejorar la calidad del agua y que no perjudique así el cultivo de nogal pecanero. Se considera que el agua de riego en el sur del estado de Chihuahua, es de buena calidad para la producción de nuez pecanera en la mayoría de sus pozos. No obstante, se sugiere tener un monitoreo periódico de las concentraciones de $\mathrm{Pb}, \mathrm{Ni}, \mathrm{As}$ y B, así como del análisis de la CE. Lo anterior debido a que la evidencia de esta investigación sugiere la posibilidad de mostrar perjuicios a la población de comunidades circundantes en la región por la merma en la calidad del agua de uso humano, además de una futura afectación para la actividad agrícola en caso del incremento de estas concentraciones.

\section{REFERENCIAS}

Arain M.B., Ullah I., Niaz A., Shah N., Shah A., Hussain Z., Tariq M., Afridi H.I., Baig J.A. y Kazi T.G. (2014). Evaluation of water quality parameters in drinking water of district Bannu, Pakistan: Multivariate study. Sustainability of Water Quality and Ecology 3-4, 114-123.

DOI: $10.1016 /$ j.swaqe.2014.12.005

ATSDR (2009). Resúmenes de Salud Pública. Plomo. Agencia para Sustancias Tóxicas y el Registro de Enfermedades [en línea]. http://www.atsdr.cdc.gov/ es/phs/es_phs13.html 07/06/2015

Ayers R.S. y Westcot D.W. (1985). Water quality for agriculture. Food and Agriculture Organization of the United Nations [en línea]. FAO Irrigation and Drainage Paper 29 Rev. 1, ISBN 92-5-102263-1 Roma, Italia. http://www.fao.org/DOCReP/003/T0234e/T0234E00. htm 15/04/2016

Bañuelos G.S., Ajwa H.A., Cáceres L. y Dyer D. (1999). Diferencias de acumulación y tolerancia a altas concentraciones de boro de especies agrícolas en el norte de Chile y California, Estados Unidos. IDESIA 17, 31-40.

Bauer M., Fulda B. y Blodau C. (2008). Groundwater derived arsenic in high carbonate wetland soils: Sources, sinks, and mobility. Sci Total Environ. 401, 109-120. DOI: $10.1016 /$ j.scitotenv.2008.03.030

Can-Chulim A., Ramírez-Ayala C., Ortega-Escobar M., Trejo-López C. y Cruz-Díaz J. (2008). Evaluación de la relación de adsorción de sodio en las aguas del río Tulancingo, estado de Hidalgo, México. Terra Latinoamericana 26, 243-252.

CONAGUA (2013). Ley Federal de Derechos. Disposiciones aplicables en materia de aguas nacionales 2013, Comisión Nacional del Agua. Ley. Tlalpan, Ciudad de México, México, 94 pp. 
García A. (2012). Criterios modernos para la evaluación de la calidad del agua de riego. IAH 7, 27-36.

Gebreyohannes F., Gebrekidan A., Hadera A. y Estifanos S. (2015). Investigations of physico-chemical parameters and its pollution implications of Elala river, Mekelle, Tigray, Ethiopia. MEJS. 7, 240-257. DOI: $10.4314 /$ mejs.v7i2.7

Grageda Grageda J., Sabori Palma R., Valenzuela Martínez A., Quijada Flores A., Núñez Moreno J.H. y Rodríguez J.C. (2011). Salinidad del suelo en huertas de nogal pecanero Carya illinoinensis (Wangenh.) K. Koch. Biotecnia. XIII, 22-27.

Lu Y., Song S., Wanga R., Liu Z., Meng J., Sweetman A.J., Jenkins A., Ferrier R.C., Li H., Luo W. y Wang T. (2015). Impacts of soil and water pollution on food safety and health risks in China. Environ Int. 77, 5-15. DOI: $10.1016 /$ j.envint.2014.12.010

Maas E. V. (1986). Salt tolerance of plants. Appl. Agric. Res. 1, 12-15.

Miyamoto S. (2004). Orchard management under water quality constraints. Memorias. 38th Western Pecan Conference Proceedings. Las Cruces, Nuevo México, EUA, pp. 71-82.

Miyamoto S. y Nesbitt M. (2011). Effectiveness of soil salinity management practices in basic-irrigated pecan orchards. Horttechnology 21, 569-576.

Miyamoto S. (2015). Salinity impacts on pecan trees. Texas agricultural experimental station. Texas A\&M AgriLife Research and USDA-NIFA, 1 pp. [en línea]. http://elpaso.tamu.edu/files/2015/03/012-SM-SalinityImpacts-on-Pecan.pdf 20/06/2015

Ojeda-Barrios D. L., Abadía J., Lombardini L., Abadía A. y Vázquez S. (2012). Zinc deficiency in field-grown pecan tres: changes in leaf nutrient concentrations and structure. J Sci Food Agri. 92, 1672-1678.

DOI: $10.1002 /$ jsfa.5530

Ojeda-Barrios D. L., Perea-Portillo E., Hernández-Rodríguez A., Ávila-Quezada G., Abadía J. y Lombardini L. (2014). Foliar fertilization with zinc in pecan trees. HortScience 49, 562-566.

Ojeda-Barrios D., Abadía J., Lombardini L., Abadía A. y Vázquez S. (2012). Zinc deficiency in field-grown pecan trees: changes in leaf nutrient concentrations and structure. J Sci Food Agr. 92, 1672-1678.

DOI: $10.1002 /$ jsfa.5530

Olmos-Márquez M. (2011). Remoción de arsénico del agua por fitorremediación con Eleocharis macrostachya en humedales construidos de flujo subsuperficial. Tesis de Doctorado. Centro de Investigación en Materiales Avanzados, S.C. (CIMAV). Chihuahua, Chihuahua, México, 99 pp.

Páez-Sánchez A., Alfaro-Cuevas-Villanueva R., CortésMartínez R. y Segovia N. (2013). Arsenic content and physicochemical parameters of water from wells and thermal springs at Cuitzeo Lake Basin, Mexico. IJIRSET. 2, 7731-7740.

Patil P.N., Sawant D.V. y Deshmukh R.N. (2012). Physicochemical parameters for testing of water - A review. IJES 3, 1194-1207. DOI: 10.6088/ijes.2012030133028

Pérez-León J.M. (2011). Manual para determinar la calidad del agua para riego agrícola. Universidad Veracruzana, Facultad de Ciencias Agrícolas. Xalapa de Enriquez, Veracruz, México, $41 \mathrm{pp}$.

Phocaides A. (2000). Technical handbook on pressurized irrigation techniques. Food and Agriculture Organization of the United Nations. Roma, Italia, 195 pp.

Rajendran A. y Mansiya B. (2015). Physico-chemical analysis of ground water samples of coastal areas of south Chennai in the post-Tsunami scenario. Ecotoxicol. Environ. Saf. 121, 218-222.

DOI: 10.1016/j.ecoenv.2015.03.037

Rhoades J.D. y Loveday J. (1990). Salinity in irrigated agriculture. Agron Monogr. 30, 1089-1142.

Sammis T., Gutschick V., Wang J. y Miller D.R. (2013). Model of water and nitrogen management in pecan trees under normal and resource-limited conditions. Agr. Water Manage. 124, 28-36.

DOI: $10.1016 /$ j.agwat.2013.03.012

SIAP (2013). Cierre de la producción agrícola por cultivo. Servicio de Información Agroalimentaria y Pesquera [en línea]. http://www.siap.gob.mx/cierre-de-la-produccion-agricola-por-estado/ 30/07/2015.

SSA (1994). Norma Oficial Mexicana NOM 127 SSA11994. Salud ambiental, agua para uso y consumo humano-límites permisibles de calidad y tratamientos a que debe someterse el agua para su potabilización. Secretaría de Salud Ambiental. Diario Oficial de la Federación. 22 de noviembre de 2000.

Valdez-Gascón B., Vieira de Figueiredo F., Ortiz-Enríquez J., Velázquez D. y Zárate-Rodríguez B. (2013). Consideraciones técnicas en el manejo del agua en huertas de nogal con riego por goteo en la costa de Hermosillo. Memorias. XIV Simposio Internacional de Nogal Pecanero. Hermosillo, Sonora, México, Septiembre 2013, pp. 36-41.

Vargas Piedra G. y Arreola Ávila J.G. (2008). Respuesta del nogal pecanero (Carya illinoensis K. Koch) a las aplicaciones foliares de nutrimentos. RCHSZA 7, 7-14.

Wagle P., Smith M.W., Wood B.W. y Rohla C.T. (2011). Response of young bearing pecan trees to spring foliar nickel applications. J. Plant Nutrition. 34, 1558-1566. DOI: $10.1080 / 01904167.2011 .585210$

Wells M.L., Conner P.J., Funderbrk J.F. y Price J.G. (2008). Effects of foliar applied boro non fruit tetention, fruit quality, and tissue boron concentration of pecan. HortScience 43, 696-699 
Wilcox L.V. y Durum W.H. (1967). Quality of irrigation water. Irrigation of Agric. Lands S. Agronomy. 11, 104-120.

Wood B.W., Reilly C.C. y Nyczepir A.P. (2004b). Mouseear of pecan: a nickel deficiency. HortScience 39, 1238-1242.
Wood B.W., Reilly C.C. y Nyczepir A.P. (2004a). Mouseear of pecan: Influence of nutrient application. HortScience 38, 95-100.

Wood B.W., Reilly C.C. y Nyczepir A.P. (2006). Field deficiency of nickel in trees: symptoms and causes. Acta Hortic. 721, 87-93.

DOI: 10.17660/ActaHortic.2006.721.10 